\title{
Das COMET-Projekt COMMBY - Aufbau eines fachübergreifenden Forschungsnetzwerkes zur Nutzung metallhältiger Nebenprodukte
}

\author{
Jürgen Antrekowitsch', Gustav Hanke², Helmut Flachberger ${ }^{3}$ und Frank Melcher $^{2}$ \\ 'Lehrstuhl für Nichteisenmetallurgie, Montanuniversität Leoben, Leoben, Österreich \\ ${ }^{2}$ Lehrstuhl für Geologie und Lagerstättenlehre, Montanuniversität Leoben, Leoben, Österreich \\ ${ }^{3}$ Lehrstuhl für Aufbereitung und Veredlung, Montanuniversität Leoben, Leoben, Österreich
}

Eingegangen 6. Juli 2020; angenommen 13. Juli 2020; online publiziert 5. August 2020

\begin{abstract}
Zusammenfassung: Recycling spielt seit langem in vielen Bereichen eine nicht mehr wegzudenkende Rolle. In der Metallurgie ist vor allem die Wiederverwertung von Schrotten weit verbreitet und neben primären Erzen eine wichtige Quelle für viele Metalle. Eine alternative Rohstoffressource stellen aber auch Nebenprodukte, welche in allen Metallherstellungsrouten anfallen, dar. In vielen Fällen werden diese jedoch nicht verarbeitet, wodurch oft beachtliche Mengen an gewinnbaren Wertstoffen verloren gehen. AuBerdem stellt die Deponierung dieser Reststoffe Firmen vor große Probleme, da sie häufig als gefährlicher Abfall eingestuft werden, was mit erhöhten Auflagen und Kosten einhergeht. Da diese Materialien sich aber aufgrund ihrer Entstehung teils erheblich von primären Erzen unterscheiden, ist eine Evaluierung des Materials und das Einbringen in bestehende Prozesse oft schwierig beziehungsweise nicht möglich. Erschwerend kommt hinzu, dass es für sekundäre Rohstoffe keine Beurteilungsverfahren gibt, wie sie für primäre Lagerstätten seit langem üblich sind. Solche Verfahren sind ein wesentlicher Faktor, um Firmen und Investoren Sicherheit in Bezug auf die Beurteilung dieser Ressourcen zu geben.
\end{abstract}

Das Projekt "COMMBY" wurde an der Montanuniversität Leoben ins Leben gerufen, um die Verwertung von metallurgischen Nebenprodukten zu forcieren. In Zusammenarbeit mit sieben Industriepartnern werden verschiedene Reststoffe beurteilt und entsprechende Prozesse entwickelt und optimiert. Ein wichtiges Ziel dieses Projekts ist es, ein Beurteilungsschema für sekundäre Rohstoffe zu entwickeln. Dieses Schema wird im Aufbau prinzipiell jenem

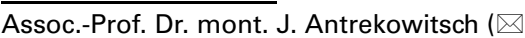
Lehrstuhl für Nichteisenmetallurgie, Montanuniversität Leoben,

Franz-Josef-Straße 18

8700 Leoben, Österreich

juergen.antrekowitsch@unileoben.ac.at
}

für primäre Rohstoffe ähneln, allerdings an die besonderen Eigenschaften und Herausforderungen von metallurgischen Reststoffen angepasst sein.

Diese Veröffentlichung stellt die Hintergründe des Projektes COMMBY im Überblick vor und präsentiert die Schwerpunkte "Charakterisierung”, "Evaluierung von Halden" und „Evaluierung der Verfahrenstechnik“. Anhand von Beispielen bisher erfolgter Arbeiten werden einige Besonderheiten und Herausforderungen in der Evaluierung metallurgischer Nebenprodukte aufgezeigt.

Schlüsselwörter: Nebenprodukte, Zertifizierung, Metalle, Charakterisierung

The COMET-Project COMMBY-Development of an Interdisciplinary Research-Network for Efficient Utilization of Metal Containing By-Products

Abstract: Recycling plays an important role in many different fields of daily life. In metallurgy, especially the recycling of scrap is very common and, beside primary ores, a major source for specific metals. However, byproducts formed during metal production are hardly used as secondary resources, even though they often bear considerable amounts of different metals. Thereby, a lot of valuable metals are dumped. Furthermore, dumping of such materials is challenging, as it is often classified as hazardous, causing higher landfilling costs. Metallurgical residues are, due to their origin, very different to primary ores. This makes the evaluation and implementation in existing processes often difficult or even impossible. There is no guideline for evaluation available, in contrast to codes used for primary raw materials. Such certification procedures are of highest importance in order to give companies and investors reliable information concerning the potential of a secondary resource. 
At Montanuniversität Leoben the COMMBY project intends to facilitate the use of by-products as secondary resources. Together with seven industrial partners, different residues are evaluated and processes for treatment are developedand optimized. A superior aim is to create an evaluation scheme for secondary resources. This guideline will be comparable to the existing ones for primary materials, but adapted for the special properties and challenges of metallurgical by-products.

This paper presents the background of the COMMBY project and summarizes the focuses "characterisation", "evaluation of dumps" and "evaluation of process technology". On the basis of some examples, peculiarities and challenges of evaluating metallurgical by-products are presented.

Keywords: By-products, Certification, Metals, Characterization

\section{Einleitung}

Nebenprodukte der metallproduzierenden Industrie werden täglich weltweit in großen Mengen produziert. Unabhängig davon, welcher Prozess angewandt wird, fallen stets mehrere unterschiedliche Rückstände an. Bei pyrometallurgischen Prozessen sind dies beispielsweise Schlacken und Stäube, bei hydrometallurgischen Prozessen Laugungsrückstände und Fällungsprodukte. Diesen metallurgischen Prozessen vorgeschaltet sind die im Wesentlichen physikalische Unterschiede nutzenden Prozesse der Aufbereitung, bei denen ebenfalls unterschiedliche Reststoffe, sogenannte Berge (Tailings), anfallen. Da in vielen Prozessschritten Komponenten verändert oder neu gebildet werden, ergibt sich eine im Vergleich zum Ausgangsmaterial (Erz) deutlich größere Komplexität in der Zusammensetzung und vor allem im Aufbau entsprechender Rückstände.

Diese Materialien werden häufig als wertlos eingestuft, enthalten jedoch sehr oft Metallkonzentrationen von wirtschaftlichem Interesse (Abb. 1; [1-3]). In vielen Fällen überschreiten die Metallgehalte sogar jene von primären Erzen. Die Gehalte ergeben sich einerseits aus technisch nicht gewinnbaren Anteilen des Hauptmetalls und andererseits

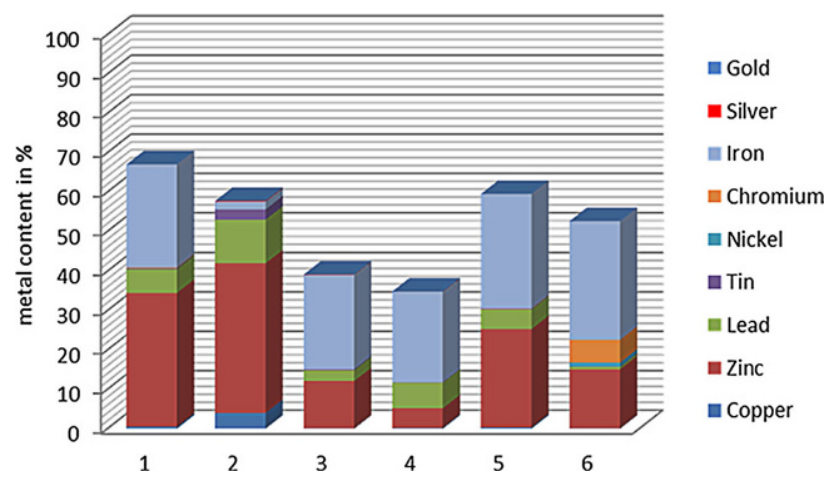

Abb. 1: Beispiele für Metallgehalte in Nebenprodukten: 1Stahlwerksstäube; 2Stäube der Kupferindustrie; 3Schlacken der Bleiindustrie; 4Fällungsrückstände der Zinkindustrie; 5 Gießereistäube; 6 Stäube der Edelstahlindustrie aus „prozessfremden“ Metallen, deren Gewinnung im primären Prozess aus verschiedensten Gründen nicht in Erwägung gezogen wurde.

Im Hinblick auf den stetigen Anstieg des Bedarfs an Metallen und der gleichzeitig größer werdenden Problematik bei der Deponierung erscheint eine möglichst vollständige Nutzung aller Nebenprodukte naheliegend und zukunftsweisend. Auch aus energetischer Sicht muss diese sekundäre Rohstoffquelle in Betracht gezogen werden, da beispielsweise Aufwände für den Bergbau und zum Teil auch für die Aufbereitung entfallen. Rückstände der Metallproduktion sind oft als "gefährlich" eingestuft. Das erschwert die Verbringung erheblich und zwingt Firmen großen Aufwand zu betreiben, um diese zu deponieren. In einem besonderen Fall werden beispielsweise eigens riesige Kavernen im Festgestein des umliegenden Gebirges angelegt, um den produzierten Fällungsrückstand zu deponieren [4]. Aus anderen Betrieben ist bekannt, dass wertvoller und wirtschaftlich aufzubereitender Rückstand mit einem nahezu wertlosen Nebenprodukt vermengt wird, um diesem einen minimalen Wert und damit die Chance auf Verwertung zu verleihen. Zusätzlich zu den neu generierten Halden findet sich noch eine Vielzahl an historischen Halden. Diese sind, aufgrund der häufig weniger effizienten Produktionsmethoden der Vergangenheit, oftmals noch reicher an Wertmetallen. Andere Halden wiederum sind aus Sicht des Wertinhaltes eher unwirtschaftlich, aber aufgrund umwelttechnischer Überlegungen kritisch. Dabei kann das Gewinnen von Metallen zumindest zur Kostendeckung beitragen.

Oft ist das Potenzial dieser Reststoffe gar nicht bekannt. Manche werden zwar bereits aufgearbeitet, jedoch oft mit veralteten Methoden, die nur einen Teil der Wertmetalle gewinnen und wiederum große Mengen an problematischen Rückständen generieren. Das größte Problem bei der Verwertung dieser Reststoffe ergibt sich aus deren besonderer Beschaffenheit. Standardverfahren der Aufbereitung sind begrenzt einsetzbar und damit eine wirtschaftlich attraktive Aufkonzentrierung nicht möglich. In weiterer Folge ist damit auch eine metallurgische Verarbeitung wirtschaftlich schwierig realisierbar, wenn überhaupt ein Prozess existiert, in den das Material sinnvoll eingebracht werden kann.

Erschwerend kommt weiters hinzu, dass es für die Evaluierung von potenziellen sekundären Lagerstätten keine Evaluierungsschemen gibt, wie sie für primäre Lagerstätten seit langem üblich sind. Für primäre Lagerstätten wurden entsprechende Zertifizierungsverfahren entwickelt, um illegale Aktivitäten (wie gezielte Fehlbeurteilungen) möglichst zu unterbinden. Den Firmen und Investoren kann so mehr Sicherheit in Bezug auf eine realistische Beurteilung von Lagerstätten gegeben werden. International bedeutende Beispiele derartiger sogenannter "Codes" sind JORC und NI 43101. Sie sind jedoch für potenzielle sekundäre Lagerstätten kaum anwendbar, da diese in vielerlei Hinsicht deutlich andere Eigenschaften aufweisen. Beispielsweise ist die Geologie und Bergbautechnik bei primären Erzen von entscheidender Bedeutung, während beide bei sekundären Lagerstätten nur von geringer Relevanz sind und vor allem die Prozesstechnik in den Bereichen „Aufbereitung" und "Metallurgie" in den Vordergrund rückt. 


\section{COMMBY}

An der Montanuniversität Leoben wurde ein fachübergreifendes Konsortium ins Leben gerufen, welches sich mit der Beurteilung und Verarbeitung von metallurgischen $\mathrm{Ne}$ benprodukten beschäftigt. Das Netzwerk, welches in diesem Projekt gebildet wurde, setzt sich aus den Lehrstühlen "Nichteisenmetallurgie", "Geologie und Lagerstättenlehre" sowie "Aufbereitung und Veredlung" der Montanuniversität Leoben und sieben internationalen Partnern aus der Industrie zusammen. Für alle sieben Firmenpartner sind metallurgische Reststoffe Teil des täglichen Geschäftes, sei es als Produzent, Verwerter oder Prozessentwickler. Durch die Bündelung aller nötigen Kompetenzen von der Evaluierung eines Materials bis hin zur metallurgischen Verarbeitung sollen für unterschiedliche Reststoffe Möglichkeiten der Verwertung gefunden werden. Übergeordnetes Ziel ist es, die gewonnenen Erkenntnisse dazu zu nutzen, ein allgemein anwendbares Beurteilungsverfahren für Reststoffe aus der metallproduzierenden Industrie zu entwickeln.

Durch die gängige Trennung der Fachgebiete "Geologie", "Aufbereitung “ und "Metallurgie" sind diese stark in ihren Bereichen spezialisiert. Dieser Vorteil kann allerdings auch zu unzureichender Zusammenarbeit außerhalb des eigenen Kompetenzbereiches führen. Die Kooperation in einem gemeinsamen Projekt wirkt dem entgegen und garantiert kurze Kommunikationswege und damit effiziente Arbeit über einzelne Fachgebiete hinaus. Die Industriepartner liefern dabei nicht nur relevante Reststoffe, sondern auch wertvolles Detailwissen in Hinblick auf deren Entstehung und Möglichkeiten der Verwertung. Ihr Mitwirken ermöglicht praxisnahe Forschung und Entwicklung, welche rasch in die Industrie übertragbar ist.

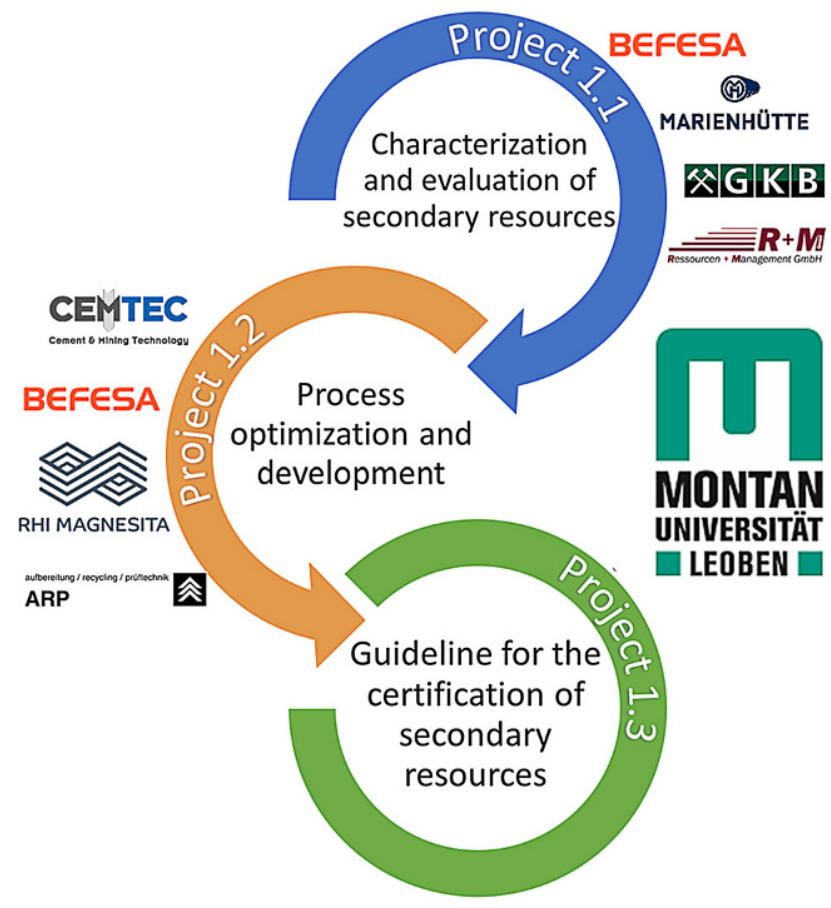

Abb. 2: COMMBY gliedert sich in drei Subprojekte, in denen die sieben Industriepartner ihre Kompetenzen einbringen
COMMBY gliedert sich dabei in drei Hauptprojekte, dargestellt in Abb. 2:

- Charakterisierung und Evaluierung

- Prozess- und Produktentwicklung und Optimierung

- Entwicklung einer Beurteilungsrichtlinie für sekundäre Rohstoffe

Die ersten beiden Teilprojekte richten sich dabei vor allem auf Rückstände, für die es seitens der Industriepartner aktuell ein großes Interesse der Aufarbeitung gibt. Einige dieser Reststoffe werden zurzeit nicht oder mit veralteten, oft wenig zufriedenstellenden Methoden aufgearbeitet. Der Fokus der in COMMBY untersuchten Nebenprodukte liegt auf zink-, blei-, kupfer- und edelmetallhaltigen Rückständen, die in Form von Stäuben, Schlämmen oder Schlacken anfallen. Die Hauptvertreter sind in der nachfolgenden Auflistung inklusive spezifischem Anfall (wo bekannt) angeführt.

- Stahlwerkstäube (15-25 kg/t),

- Rückstände aus der primären Zinkproduktion (600$900 \mathrm{~kg} / \mathrm{t}$ ),

- Gießereistäube (10-20 kg/t),

- Stäube der Kupferindustrie (20-60 kg/t),

- Schlacken der Bleiindustrie (600-1000 kg/t) und

- Berge der Aufbereitungsindustrie.

Nimmt man nur einige dieser Reststoffe und errechnet den Wert an enthaltenen Metallen, die mangels Aufarbeitung jährlich deponiert werden, erreicht man rasch Euro-Beträge in Milliardenhöhe, was einmal mehr das große Potenzial dieser Nebenprodukte verdeutlicht.

Der dritte Teilbereich „Entwicklung einer Beurteilungsrichtlinie" nutzt die Expertise aus den ersten beiden Teilprojekten und ermöglicht damit die Erarbeitung eines Zertifizierungsschemas für Nebenprodukte, welches eine Beurteilungsbasis für Investoren und interessierte Firmen bildet. Dies wird durch internationale Experten - sogenannte "Competent Persons" - unterstützt, die im Bereich der Zertifizierung bereits ausreichend Erfahrung aus dem primären Bereich besitzen und damit zur Erstellung eines geeigneten Schemas beitragen.

\section{Charakterisierung}

Soll ein Reststoff aufgearbeitet werden, so ist es von entscheidender Bedeutung, alle wesentlichen Charakteristiken zu kennen. Nur so kann eruiert werden, welche Art der Verarbeitung geeignet ist, beziehungsweise worauf ein neu entwickelter Prozess ausgelegt sein muss. Der erste Schritt ist immer eine Gesamtanalyse, um das theoretisch wirtschaftliche Potenzial aufzuzeigen. Ergibt sich hierbei, dass nennenswerte Gehalte an Wertmetallen vorhanden sind, müssen folgende Fragen geklärt werden, um die Nutzbarkeit realistisch beurteilen zu können und passende Aufarbeitungsmethoden zu wählen beziehungsweise zu entwickeln: 
Abb. 3: Beispiel eines Charakterisierungsschemas für metallurgische Nebenprodukte

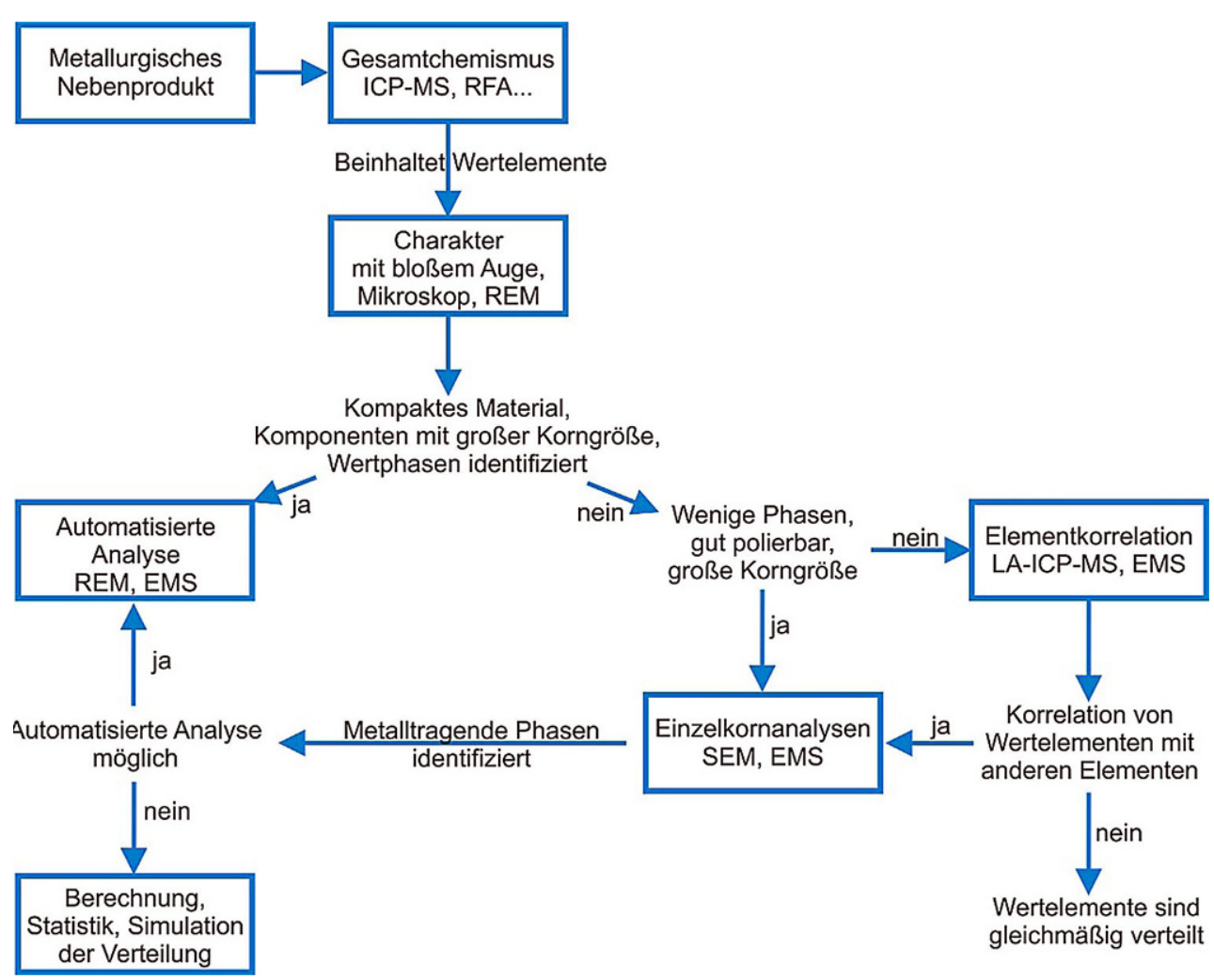

- Welche Phasen beinhalten die gefragten Elemente (Wertmetalle und Störstoffe)?

- Wie bedeutend sind die identifizierten Trägerphasen bezüglich ihrer Quantität?

- Wie ist die Morphologie der bedeutenden Komponenten (Größe, Form ....)?

- Wie verwachsen liegen diese Phasen vor?

Da es sich bei metallurgischen Nebenprodukten häufig um sehr feinkörniges Material handelt, stellt vor allem die Korngröße eine besondere Herausforderung dar. Abb. 3 zeigt ein grundlegendes Charakterisierungsschema, wie es im Speziellen für entsprechende Nebenprodukte Anwendung finden soll [5]. Am Beginn steht, wie oben erwähnt, eine chemische Gesamtanalyse. Diese ist in den meisten Fällen mittels Standardverfahren wie zum Beispiel RFA (Röntgenfluoreszenzanalyse) und ICP-MS (Massenspektrometrie mit induktiv gekoppeltem Plasma) zu bewerkstelligen. Für die Identifizierung und Quantifizierung von Phasen ist es entscheidend, welche Methoden überhaupt anwendbar sind. Diese sind vor allem durch die Korngröße des Materials, beziehungsweise durch die Vergrößerung des Mikroskops beschränkt. Röntgendiffraktometrie eignet sich grundsätzlich für die Identifizierung der Hauptkomponenten. Ihr großer Vorteil ist, dass die Korngröße keine entscheidende Rolle spielt. Rasterelektronenmikroskope (REM) sind in vielerlei Hinsicht das Mittel der Wahl zur Charakterisierung von feinkörnigem Material bis zu einer Korngröße von einigen Mikrometern. Neben der morphologischen Beschreibung der Partikel ist auch eine Phasenbestimmung mittels (semi-)quantitativer chemischer Messung möglich.
Elektronenstrahlmikrosonden (EMS), ausgerüstet mit wellenlängendispersiven Röntgenspektrometern, erlauben genauere Messungen mit deutlich niedrigeren Nachweisgrenzen. Wenn das Material genügend Voraussetzungen erfüllt (eine gewisse Korngröße aufweist, gut polierbar ist und die Wertphasen bekannt sind), können automatisierte Methoden der Phasenquantifizierung angewandt werden. Entsprechende Programme sind für Licht- und Elektronenmikroskope weit verbreitet. Diese stellen jedoch hohe Ansprüche an Korngröße und Probenpräparation. Werden diese nicht erfüllt, müssen Phasen manuell charakterisiert und quantifiziert werden. Bei Elementen, die in geringer Quantität vorhanden, aber aufgrund ihres Preises trotzdem relevant sind (wie zum Beispiel Silber), sind Trägerphasen unter Umständen nicht lokalisierbar. In diesem Fall können geochemische Mappingverfahren mit möglichst geringer Ortsauflösung eingesetzt werden. Dabei werden Profillinien oder Raster bestehend aus vielen Messpunkten mit LAICP-MS (Laser-Ablation verbunden mit induktiv gekoppelter Plasma-Massenspektrometrie) oder Elektronenstrahlmikrosonde auf feinkörnigen verpressten und polierten Proben gemessen. Durch das Volumen des angeregten Materials im Bereich von einigen $\mu \mathrm{m}^{3}$ bis einigen hundert $\mu \mathrm{m}^{3}$ werden Mischanalysen erzeugt, deren Zusammensetzung von der Verteilung unterschiedlicher Phasen im angeregten Volumen abhängt. Korrelationen bestimmter Elemente in solchen Mikrovolumina erlauben Rückschlüsse auf Trägerphasen und deren quantitative Relevanz. Korreliert beispielsweise das häufig vorkommende Blei mit dem seltenen Silber positiv, weist das auf Bleiphasen hin, die als Silberträger dienen. Korreliert Silizium negativ mit allen 
Wertmetallen, sind Silikate offensichtlich keine relevanten Trägerphasen.

Als Beispiel der Anwendung des Schemas ist im Folgenden die Untersuchung von Laugungsrückständen der Zinkindustrie (Jarosit) angeführt.

Zink wird heutzutage fast ausschließlich über einen hydrometallurgischen Prozess gewonnen. Dabei wird das sulfidische Sphaleritkonzentrat geröstet und anschließend in mehreren Laugungsschritten in Schwefelsäure gelaugt [6] Die dabei anfallenden Rückstände stehen seit längerem im Fokus der Forschungstätigkeiten und stellen daher ein gutes Beispiel für die Evaluierung der Verwertungsmöglichkeiten dar [7]. Mehrere unterschiedliche Jarositrückstände wurden im Detail charakterisiert. Chemische Analysen zeigten durchwegs beachtenswerte Gehalte an Blei, Zink und Silber, untergeordnet auch Kupfer und in manchen Fällen Gold. Bezugnehmend auf die oben angeführten Fragen werden im Folgenden die wichtigsten Charakteristiken auszugsweise beschrieben:

Als eindeutige Blei- und Zinkphasen konnten Bleisulfid, Bleisulfat, Bleioxid, Zinksulfid und Zinkoxid identifiziert werden. Es stellte sich auch heraus, dass das Mineral Jarosit (welches den größten Teil des Rückstandes ausmacht) ebenfalls Blei und Zink einbaut.

Da das Mineral Jarosit im Rückstand dominant ist, sind alle anderen blei- und zinkführenden Phasen von untergeordneter Bedeutung.

Die Korngröße ist durchwegs sehr fein mit ca. 95\% kleiner $100 \mu \mathrm{m}$ und $60 \%$ kleiner $10 \mu \mathrm{m}$. Die Morphologie weist keine technisch bedeutenden Besonderheiten (z. B. Kornform) auf.

Jarositpartikel zeigen häufig eine Zonierung. Diese Zonen unterscheiden sich meist in ihren Zink- und Bleigehalten. Außerdem kommen Silber-Kupfer-Einschlüsse in Quarzen und Feldspäten häufig vor.

Insgesamt können für eine Verarbeitung folgende Schlüsse gezogen werden:

- Methoden der Aufbereitung sind wenig erfolgversprechend, da die Wertmetalle in allen quantitativ relevanten Phasen eingebunden sind. Die intensive Verwachsung würde die Aufbereitung dieses Reststoffes deutlich erschweren.

- Ein hydrometallurgischer Prozess müsste aufgrund der Komplexität des Materials und damit der Vielzahl an Phasen, die gelaugt werden müssen, ebenfalls sehr komplex gestaltet sein.

- Die Anwendung eines pyrometallurgischen Prozesses, bei dem sämtliche Elemente in einem zweistufigen Prozess gewonnen werden können, scheint am vielversprechendsten.

- Für die Verwertung in einem pyrometallurgischen Prozess muss das Material getrocknet und pelletiert werden. Die geringe Korngröße würde die Handhabung deutlich erschweren und zu einem hohen Austrag (Verlust) mit dem Abgas führen.

- Die Temperatur für die Kalzinierung sollte zwischen 800 und $900^{\circ} \mathrm{C}$ liegen, um den Schwefel zu entfernen, ohne jedoch Wertmetalle zu verflüchtigen.

\section{Evaluierung von Reststoffhalden}

Wie auch im Falle von primären Ressourcen, spielt die Evaluierung der Lagerstätte eine bedeutende Rolle. Wenn das jeweilige Nebenprodukt nicht unmittelbar nach dem Anfall im Betrieb einer Verwertung zugeführt werden soll, endet es auf Halden. Diese unterscheiden sich in Art und Aufbau je nach Gefährlichkeit des Materials sowie der lokalen Gesetzgebung. Die Evaluierung einer derartigen anthropogenen (sekundären) Lagerstätte gestaltet sich jedoch völlig anders, als dies bei primären Lagerstätten der Fall ist. Die wesentlichsten Charakteristiken von sekundären Halden im Vergleich zu primären Erzlagerstätten sind:

- Die horizontale Ausdehnung ist zumeist sehr genau bekannt, häufig auch die vertikale.

- Je nach Alter gibt es Hinweise auf das deponierte Material bzw. die deponierten Materialien.

- Es handelt sich überwiegend um Nebenprodukte, welche zumindest einen oder auch mehrere Aufbereitungsschritte durchlaufen haben.

- Besonders im Falle von Stäuben ist die Morphologie aufgrund der Entstehungsgeschichte überaus komplex, Ähnliches gilt für Fällungsprodukte.

- Durch die zumeist erfolgte einfache Schüttung ist die Entnahme von kompakten Bohrkernen nicht möglich. Andere Verfahren zur Probenextraktion müssen angewendet werden.

- Immer wieder finden sich auf entsprechenden Halden Fremdmaterialien, die nicht für die jeweilige Halde vorgesehen waren.

Grundsätzlich erfolgt am Beginn einer Evaluierung die Charakterisierung von ersten Proben, die möglichst repräsentativ für die Halde sind. Das heißt, dass sie nicht zu oberflächennahe bzw. an sehr exponierten Stellen entnommen werden dürfen.

Nach parallelen Evaluierungen geeigneter Verwertungsverfahren muss bei positiver Entscheidung zur weiteren Vorgehensweise eine detaillierte Beprobung der Halde mit Hilfe eines Bohrrasters erfolgen. Die bereits zur Verfügung stehenden Informationen, wie Analysen zum Zeitpunkt der Verhaldung, bzw. Daten aus früheren Beprobungen sowie der Materialtyp, entscheiden dabei über die Anzahl der Proben und die Rasterdichte.

Zu den wichtigsten Ergebnissen einer entsprechenden Beprobung zählen:

- Homogenität/Heterogenität der Halde

- Typ und Position etwaiger Fremdstoffe

- Verteilung der Wertmetalle

- Verteilung der Verunreinigungskomponenten

- deponierte Menge

- Korngrößenverteilungen

Nachfolgend ist ein Evaluierungsprozess am Beispiel einer Schlackenhalde der Bleigewinnung in Südosteuropa beschrieben.

Schlacken der Bleiindustrie enthalten häufig Zink, welches gemäß Literatur bereits ab etwa $8 \%$ noch in der 
Abb. 4: Lageplan der Bohrungen auf der Schlackenhalde sowie das Bohrgerät
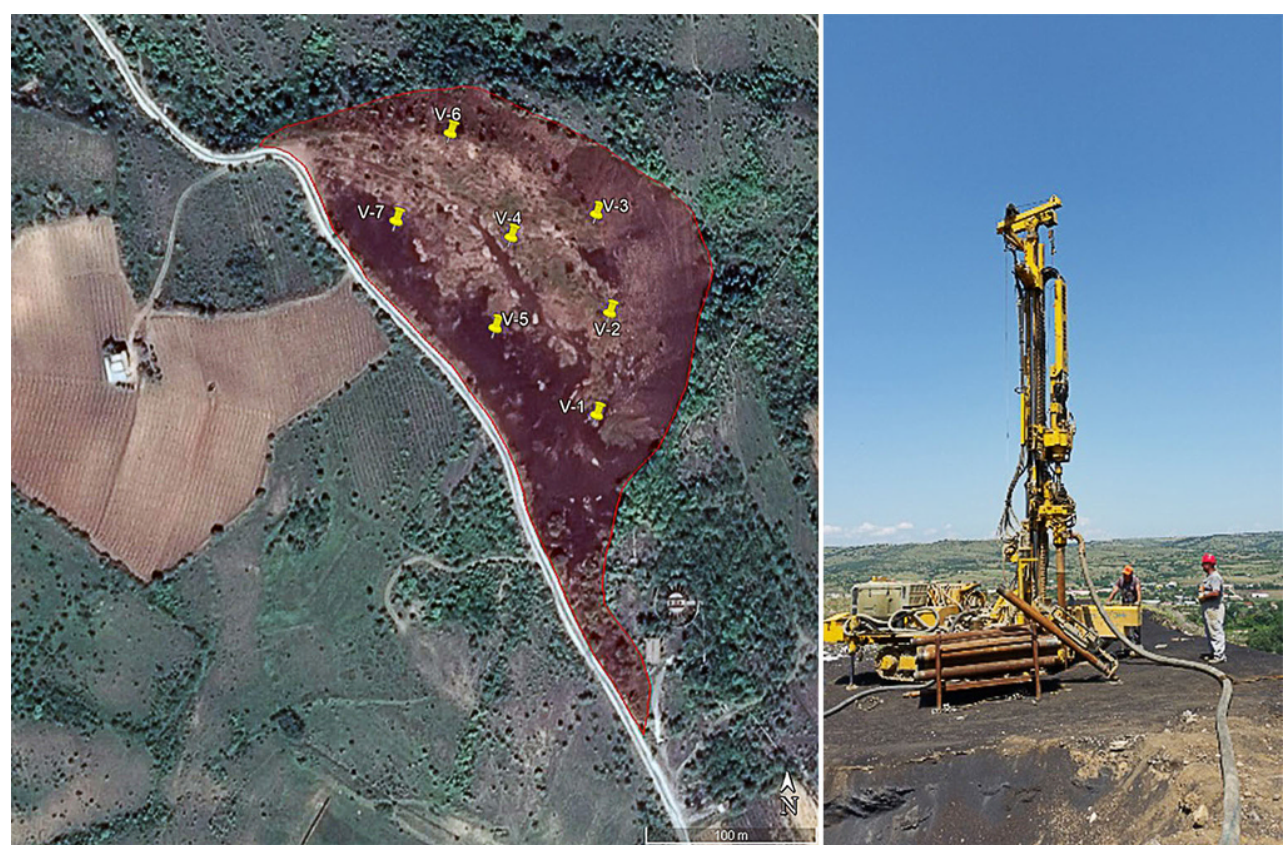

Bleihütte durch sogenanntes Verblasen gewinnbringend wiedergewonnen werden kann. Dennoch finden sich weltweit zahlreiche Halden mit Zinkgehalten deutlich über $8 \%$ Zink.

Erste Untersuchungen von Proben, unter Einbezug bereits vorhandener Daten, führten zum nächsten Schritt, der Evaluierung möglicher Verarbeitungsprozesse. Die Ergebnisse, im Speziellen der Zinkgehalt von 9\% sowie mögliche bestehende bzw. optimierbare Verfahrenskonzepte, zeigten ausreichend Potenzial, um die detaillierte Haldenbeprobung umzusetzen. Abb. 4 zeigt eine Aufnahme der Halde mit den unterschiedlichen Positionen der Bohrungen sowie das verwendete Bohrgerät.

Aufgrund der zahlreich vorhandenen Vorkenntnisse war eine vergleichsweise geringe Anzahl an Bohrungen notwendig, um zu den oben beschriebenen Erkenntnissen zu gelangen.

Dennoch ergaben die Arbeiten 80 Proben, welche im Detail analysiert und ausgewertet wurden.

Im beschriebenen Fall ergaben sich überaus zufriedenstellende Werte, die folgende generelle Rückschlüsse ermöglichten:

- Der Haldenkörper kann mit etwa 1,5 Mio. Tonnen abgeschätzt werden, was die vorangegangenen Annahmen grundsätzlich bestätigen konnte.

- Der Zinkgehalt zeigte sich als weitgehend gleichbleibend über die Halde, was eines der wichtigsten Ergebnisse darstellte.

- Unerwünschte Kontaminationen durch Fremdsubstanzen (anderer deponierter Abfall bzw. Rückstände) konnten nicht gefunden werden.

- Auch andere Details weisen auf eine weitgehende Homogenität der Halde hin.
Leider zeigen viele andere Halden in derartigen Beprobungen immer wieder starke Diskrepanzen im Wertmetallgehalt sowie häufig die Einbindung völlig fremder Reststoffe, die nichts zum Wertinhalt beitragen bzw. aufgrund von unerwünschten Elementen negative Auswirkungen haben.

Mit positiven Beispielen wie dem beschriebenen, aber besonders bei negativen Fällen zeigt sich die Wichtigkeit dieses Schrittes für eine Zertifizierung.

\section{Evaluierung der Verfahrenstechnik}

In Zertifizierungssystemen für primäre Rohrstoffe wie JORC oder NI-43101 stellt die Verfahrenstechnik lediglich einen sogenannten „Modifying Factor" dar. Dies ist nur einer von mehreren Faktoren, welcher bestimmt, inwieweit eine Ressource zu einer Reserve wird, d.h. eine wirtschaftliche Gewinnung des Metalls zum jetzigen Zeitpunkt erlaubt. Hintergrund ist die Annahme, dass für die häufigsten Minerale ein Standard-Aufbereitungsprozess sowie ein Standard-Metallgewinnungsprozess vorliegt und vielfach auch nur ein einziges zu gewinnendes Metall im Fokus steht.

Im Vergleich dazu stellt die Verfahrenstechnik für Nebenprodukte einen der wichtigsten Beurteilungsfaktoren dar, der häufig über Verwertbarkeit oder Verbleib auf der Halde entscheidet. Beispielsweise stellt der Mangel an anwendbaren, typischen Aufbereitungsverfahren wie die Flotation und damit das Fehlen eines hochkonzentrierten Ausgangsstoffes die effektive Metallgewinnung vor große Herausforderungen. Des Weiteren sind es meist mehrere Metalle, die es parallel rückzugewinnen gilt, sodass oftmals eine Kombination von unterschiedlichen Verfahren ins Auge gefasst werden muss und nicht wie bei primären Konzentraten auf eine Standardprozessabfolge zurückgegriffen werden kann. 


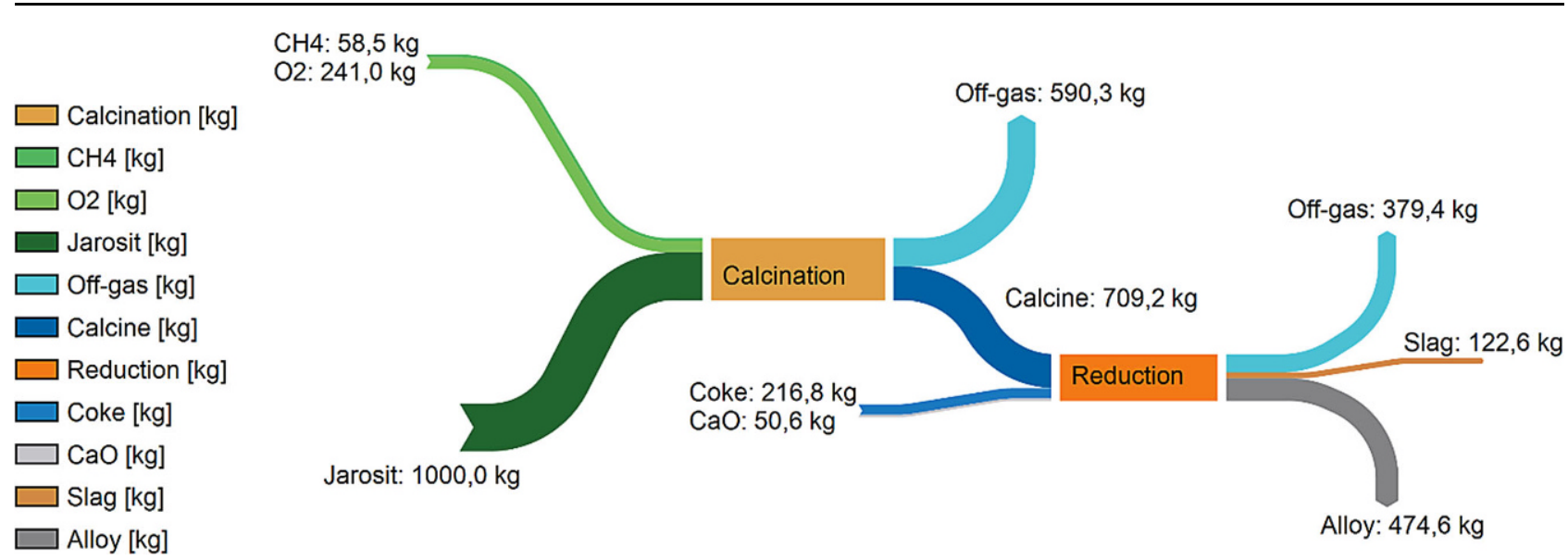

Abb. 5: Massenbilanz eines Verwertungsverfahrens für Jarosit

Beurteilt man gegenwärtig Prozesse, so betrifft dies nicht nur den physikalischen oder chemischen Vorgang oder das Aggregat selbst. Vielmehr müssen eine Reihe von Begleitfaktoren Berücksichtigung finden, die heute mitentscheidend für die wirtschaftliche Verwertung von Nebenprodukten sind.

Besondere Bedeutung kommt dem Energieaufwand in einem Metallgewinnungsprozess zu. Befindet man sich in jenem Teil der Erde, der als "westliche Welt" bezeichnet wird, wo Klimaschutz und Minimierung des $\mathrm{CO}_{2}$-Ausstoßes große Bedeutung erlangt haben, ist es wohl nur mehr schwer vorstellbar, dass noch vor wenigen Jahrzehnten der Energieaufwand in Metallgewinnungsprozessen eine deutlich geringere Rolle gespielt hat. Waren beispielsweise ausreichend Kohlenstoffträger verfügbar, war der spezifische Verbrauch pro Tonne Metall von untergeordneter Bedeutung. Der Verbrauch der doppelten bis dreifachen stöchiometrisch notwendigen Menge, um eine um wenige Prozente verbesserte Metallausbeute zu erlauben, war keine Seltenheit, Kriterien wie " $\mathrm{CO}_{2}$-Footprint" wurden vernachlässigt oder gar nicht beachtet. Leider sind diese Umstände besonders im asiatischen Raum auch heute noch gängige Praxis.

Zusammengefasst lassen sich folgende Beurteilungskriterien auflisten:

- Verfügbarkeit eines physikalisch-chemischen Prozesses zur Metallgewinnung

- Möglichkeit zur simultanen Gewinnung mehrerer enthaltener Metalle

- Ausbringen der wesentlichen zu gewinnenden Metalle

- Energieaufwand bezogen auf die Wertschöpfung

- Umweltaspekte und Arbeitssicherheit

- Komplexität und Standardisierung der Anlagentechnik

- Möglichkeit zur Entwicklung neuer bzw. Optimierung bestehender Prozesse

- Qualität der Produkte

Die Produktqualität spielt ebenfalls eine wesentliche Rolle, da aus der Aufarbeitung von Nebenprodukten zumeist nicht unmittelbar börsenfähige Metalle, sondern vielmehr
Zwischenprodukte erzeugt werden. Diese sind nicht immer einfach zu beurteilen und werden je nach Verbindung und Reinheit als Vorstoff gesehen. In der Vergangenheit wurden diese Zwischenprodukte häufig als Konzentratersatz für die primäre Metallproduktion verkauft. Solange diese Vorgehensweise ausreichend lukrativ war, entstand keine Notwendigkeit, beim Recycling weiter in die Produkttiefe bis zum reinen Metall oder marktfähigen Verbindungen vorzudringen. Heute findet man jedoch immer mehr die Forderung, die Möglichkeiten einer Produktverbesserung in Erwägung zu ziehen, um einen höheren Wert zu generieren und einen breiteren Markt vorzufinden.

Als wesentliches Werkzeug zur Beurteilung eines möglichen Prozessablaufes dient die Massen- und Energiebilanz für das zu verarbeitende Nebenprodukt. Daraus lassen sich viele der oben genannten Kriterien entsprechend ableiten.

Als Beispiel dient im Folgenden die Verarbeitung eines Laugungsrückstandes mit den zugehörigen Bilanzen (Abb. 5).

Dabei sind der Energiebedarf der einzelnen Schritte, die Zwischen- und Endprodukte sowie das Aufkommen eventuell neuer Reststoffe, Abwässer und Abgase erkennbar, woraus großteils die oben genannten Beurteilungskriterien abgeleitet werden können.

\section{Zusammenfassung}

Nebenprodukte der metallurgischen Industrie, wie Stäube, Schlacken und Schlämme, stellen eine interessante Rohstoffquelle für die Zukunft dar. Besonders in Europa zeigt sich eine hohe Verfügbarkeit durch zahlreiche Halden sowie kontinuierlich anfallende Materialien. Die Komplexität dieser Rückstände stellt vielfach eine Herausforderung hinsichtlich der Metallrückgewinnung dar. Als wesentliche Hürde auf dem Weg zur effektiven Nutzung zeigt sich das Fehlen eines Zertifizierungssystems, ähnlich jenen für primäre Ressourcen, welches interessierten Investoren oder Firmen ermöglicht, diese Rohstoffquellen auf der Basis verifizierbarer und vertrauenswürdiger Daten wirtschaftlich nutzen zu können. 
Die Montanuniversität Leoben hat mit drei Lehrstühlen und sieben Industriepartnern ein Projekt gestartet, welches sich der Erarbeitung der Grundlagen eines Zertifizierungssystems widmet. Das Projekt wird unterstützt durch die Forschungsförderungsgesellschaft FFG sowie die Bundesländer Steiermark und Burgenland. Nach den ersten eineinhalb Jahren konnten bereits wichtige Fortschritte erreicht werden.

Nur allzu oft verlassen Rohstoffe, im Speziellen metallische Schrotte, Europa und stehen für unsere Gesellschaft nicht mehr zur Verfügung. Das vorrangige Ziel des COMMBY-Projektes ist es, die wertvolle, bisher häufig ungenutzte Wertmetallquelle "Nebenprodukte" für die europäische Industrie nutzbar zu machen und damit einen wichtigen Beitrag zur europäischen Rohstoffversorgung zu liefern.

Funding. Open access funding provided by Montanuniversität Leoben.

Open Access Dieser Artikel wird unter der Creative Commons Namensnennung 4.0 International Lizenz veröffentlicht, welche die Nutzung, Vervielfältigung, Bearbeitung, Verbreitung und Wiedergabe in jeglichem Medium und Format erlaubt, sofern Sie den/die ursprünglichen Autor(en) und die Quelle ordnungsgemäß nennen, einen Link zur Creative Commons Lizenz beifügen und angeben, ob Änderungen vorgenommen wurden.

Die in diesem Artikel enthaltenen Bilder und sonstiges Drittmaterial unterliegen ebenfalls der genannten Creative Commons Lizenz, sofern sich aus der Abbildungslegende nichts anderes ergibt. Sofern das betreffende Material nicht unter der genannten Creative Commons Lizenz steht und die betreffende Handlung nicht nach gesetzlichen Vorschriften erlaubt ist, ist für die oben aufgeführten Weiterverwendungen des Materials die Einwilligung des jeweiligen Rechteinhabers einzuholen.
Weitere Details zur Lizenz entnehmen Sie bitte der Lizenzinformation auf http://creativecommons.org/licenses/by/4.0/deed.de.

\section{Literatur}

1. Balladares, E.; Kelm, U.; Helle, S.; Parra, R.; Araneda, E.: Chemicalmineralogical characterization of copper smelting flue dust. Dyna, Vol. 81 (2014), iss. 186, pp 11-18. DOI:10.15446/dyna.v81n186.32852

2. Rizescu, C.; Bacinschi, Z.; Stoian, E.; Polinescu, A.: Characterisation of steel mill electric-arc furnace dust. In: Advances in waste management. 4th WSEAS International Conference on Waste Management, Water Pollution, Indoor Climate (WWAI '10), 2010, pp. 139-143. DOI:10.1016/s0140-6701(05)80312-1

3. Jamieson, H. E.; Walker, S. R.; Parsons, M. B.: Mineralogical characterization of mine waste, Applied Geochemistry, vol. 57 (2015), pp 85-105

4. Boliden 2020. https://www.boliden.com/operations/smelters/bolidenodda, online (Abruf am 15.06.2020)

5. Hanke, G.; Onuk, P.; Antrekowitsch, J.: Characterization Strategies for Metallurgical By-Products: Case Study Jarosite, FLOGEN Sustainable Industrial Processing Summit, SIPS2018 vol. 7, Nonferrous/Mineral Processing/Environmental Protection. Montreal, Canada: FLOGEN Star Outreach, 2018

6. Sinclair, R. J.: The Extractive Metallurgy of Zinc. Victoria (Australia): Australasian Institute of Mining and Metallurgy; 2001, pp 31-105

7. Hanke, G.; Antrekowitsch, J.: Characterisation and pyrometallurgical recycling of jarosite type residues out of zinc primary metallurgy. World of Metallurgy - Erzmetall, vol. 71 (2018), iss. 1, pp 25-30

Hinweis des Verlags. Der Verlag bleibt in Hinblick auf geografische Zuordnungen und Gebietsbezeichnungen in veröffentlichten Karten und Institutsadressen neutral. 